\title{
IRREDUCIBLE INDUCED REPRESENTATIONS OF FELL BUNDLE $C^{*}$-ALGEBRAS
}

\author{
MARIUS IONESCU AND DANA P. WILLIAMS
}

\begin{abstract}
We give precise conditions under which irreducible representations associated to stability groups induce to irreducible representations for Fell bundle $C^{*}$-algebras. This result generalizes an earlier result of Echterhoff and the second author. Because the Fell bundle construction subsumes most other examples of $C^{*}$-algebras constructed from dynamical systems, our result percolates down to many different constructions including the many flavors of groupoid crossed products.
\end{abstract}

\section{INTRODUCTION}

One of the fundamental tasks in any study of the ideal structure of $C^{*}$-algebras associated to dynamical systems is to construct a suitably large class of irreducible representations. In the type I case, it makes sense to try to find representatives of all equivalence classes of irreducible representations (for example, see 20, Theorem 8.16]). In general, in the presence of suitable amenability, it is only reasonable to try to construct enough irreducible representations to account for all primitive ideals. The quintessential example is the Gootman-Rosenberg-Sauvagoet Theorem (see [20, §8.3] for a precise statement and further references): the GRS-Theorem says that for a separable $C^{*}$-dynamical system $\alpha: G \rightarrow$ Aut $A$ with $G$ amenable, every primitive ideal in $A \rtimes_{\alpha} G$ is induced in an appropriate sense from a stability group $G_{P}=\{s \in G: s \cdot P=P\}$ with respect to the induced action of $G$ on Prim $A$ for some $P \in \operatorname{Prim} A$.

Motivated in part by the GRS-Theorem and by results in the case where the action of $G$ on Prim $A$ is smooth, Echterhoff and the second author have conjectured that every separable $C^{*}$-dynamical system $(A, G, \alpha)$ satisfies the Effros-Hahn Induction Property (EHI) which asserts that if $P \in \operatorname{Prim} A$ and $J \in \operatorname{Prim}\left(A \rtimes_{\alpha} G_{P}\right)$ with Res $J=P$, then $\operatorname{Ind}_{G_{P}}^{G} J$ is a primitive ideal in $A \rtimes_{\alpha} G$. (See [2, §2] for precise definitions and additional details.) Although the validity of the conjecture is open in general - even when $G$ is amenable and the GRS-Theorem holds it was shown in [2] to hold in a wide variety of cases including all separable systems with $A$ of type I. Moreover, in all cases in which the conjecture is known to hold, a stronger property holds, called strong-EHI, which asserts that if $\rho \rtimes \pi$ is an irreducible representation of $A \rtimes_{\alpha} G_{P}$ with $\operatorname{ker} \rho=P$, then $\operatorname{Ind}_{G_{P}}^{G}(\rho \rtimes \pi)$ is an

Received by the editors May 13, 2013.

2010 Mathematics Subject Classification. Primary 46L05, 46L55.

Key words and phrases. Fell bundle, irreducible representation, ideal structure, Fell bundle $C^{*}$-algebra.

The first and second authors were supported by individual grants from the Simons Foundation.

The second author would like to thank Marius and his colleagues at Colgate for a very pleasant and productive visit. 
irreducible representation of $A \rtimes_{\alpha} G$. The key observation in [2] concerning group $C^{*}$-dynamical systems is that strong-EHI always holds if, in addition, $\rho$ is assumed to be a homogeneous representation (as defined in, for example, [20, Definition G.1]) — this is the content of [2, Theorem 1.7].

Our goal here is to extend the results on inducing irreducible representations in [2], and [2, Theorem 1.7] in particular, to other sorts of dynamical system constructions built not only on groups but on groupoids. At first glance, there are a horrifying number of potential targets for such an analysis. For example, there are groupoid $C^{*}$-algebras with or without a cocycle, and more generally, one could consider the $C^{*}$-algebras associated to twists over groupoids (also called $\mathbf{T}$-groupoids). There are also Green twisted dynamical systems, groupoid dynamical systems and even twisted versions of groupoid dynamical systems to name a few of the most important. Fortunately, as described in detail in [9, §3] or [10, §2], all these variants are subsumed using the $C^{*}$-algebra of a separable Fell bundle $p: \mathscr{B} \rightarrow G$ over a locally compact groupoid $G$ with a Haar system. In this event, the sections $A=\Gamma_{0}\left(G^{(0)} ; \mathscr{B}\right)$ form a $C^{*}$-algebra and the groupoid $G$ acts continuously on $\operatorname{Prim} A$. Any representation $L$ of $C^{*}\left(G_{P}, \mathscr{B}\right)$ is associated to a representation $\pi$ of the $C^{*}$-algebra $A$. Our main theorem (Theorem 4.1) says that if $L$ is irreducible, ker $\pi=P$ and $\pi$ is homogeneous, then $\operatorname{Ind}_{G_{P}}^{G} L$ is irreducible. This result extends [2] and we will illustrate how it "trickles down" to other dynamical systems settings in Section 6.

Our proof requires an intermediate result which is of considerable interest on its own. Namely if $p: \mathscr{B} \rightarrow G$ is a separable Fell bundle over a locally compact groupoid $G$ with Haar system, then we show that if $u \in G^{(0)}$, if $G(u)=\{x \in$ $G: r(x)=u=s(x)\}$ is the stability group of $u$ in $G$ and if $L$ is an irreducible representation of $C^{*}(G(u), \mathscr{B})$, then $\operatorname{Ind}_{G(u)}^{G} L$ is an irreducible representation of $C^{*}(G, \mathscr{B})$ (Theorem 2.1). This result is a direct generalization of [6, Theorem 5] where the result is proved for groupoid $C^{*}$-algebras (so that $\mathscr{B}$ is the trivial bundle $\mathscr{B}=G \times \mathbf{C})$. In fact the proof is disarmingly similar to that in $[6$, but extra care must be taken to account for the rather significant difference between scalar-valued sections of a trivial bundle and Banach space-valued sections of potentially highly nontrivial Fell bundles. Combining Theorem 2.1 with the usual induction in stages allows us to reduce the proof of our main theorem to the more comforting setting of a Fell bundle over a group (rather than a groupoid).

Our paper is organized as follows. We start in Section 2 with a very brief review of induced representations of Fell bundle $C^{*}$-algebras and prove our generalization, Theorem 2.1] of [6, Theorem 5]. In Section [3 we give the precise definition of the strong Effros-Hahn Induction property in the Fell bundle setting. In Section 4, we give our proof of the main theorem taking advantage of induction in stages and Theorem 2.1 to reduce to the case that $G$ is a group. In Section 5 we see that the additional hypothesis of homogeneity is automatically satisfied in the case that points are locally closed in the $C^{*}$-algebra $A=\Gamma_{0}\left(G^{(0)} ; \mathscr{B}\right)$ associated to the Fell bundle $p: \mathscr{B} \rightarrow G$. While this includes many interesting classes of algebras, it in particular applies any time $A$ is of type I. In Section [6 we examine how the Fell bundle result applies to the examples of groupoid dynamical systems and their twisted counterparts. It is worth noting that special cases of the latter include Green twisted systems in the case where $G$ is a group, and the $C^{*}$-algebras of twists or T-goupoids when $\mathscr{B}$ is a trivial line bundle. 
Assumptions. Throughout, $p: \mathscr{B} \rightarrow G$ will be a saturated, separable Fell bundle over a locally compact groupoid $G$ as defined in [10]. Thus $p: \mathscr{B} \rightarrow G$ is an upper semicontinuous Banach bundle over a second countable locally compact groupoid $G$ such that its continuous sections, vanishing at infinity on $G, \Gamma_{0}(G ; \mathscr{B})$, form a separable Banach space with respect to the supremum norm. Furthermore, all our groupoids are assumed to be second countable, locally compact and Hausdorff. When $G$ is a groupoid, it will be assumed to have a Haar system $\left\{\lambda^{u}\right\}_{u \in G^{(0)}}$. We will write $A=\Gamma_{0}\left(G^{(0)} ; \mathscr{B}\right)$ for the $C^{*}$-algebra of $\mathscr{B}$ over $G^{(0)}$. We then follow [10, §1] to make the compactly supported continuous sections $\Gamma_{c}(G ; \mathscr{B})$ into a *algebra with $C^{*}$-completion $C^{*}(G, \mathscr{B})$. When dealing with any sort of Banach bundle $p: \mathscr{B} \rightarrow X$, we will use a roman font, $B(x)$, to indicate the fibre over $x \in X$ together with its Banach space structure. When we have the need to work with a Fell bundle over a group - in particular, when we restrict a Fell bundle over a groupoid to a subgroup - we will, for the sake of consistency, treat the underlying group as a groupoid as regards our conventions with modular functions 1 (See [8, §1.5] for an elaboration on this.)

\section{INDUCING FROM $G(u)$}

In this section we prove the following generalization of [6, Theorem 5] to Fell bundle $C^{*}$-algebras.

Theorem 2.1. Suppose that $p: \mathscr{B} \rightarrow G$ is a separable Fell bundle over a locally compact groupoid with a Haar system. Let $u \in G^{(0)}$ and let $G(u):=\{x \in G$ : $r(x)=u=s(x)\}$ be the stability group at $u$. Suppose that $L$ is an irreducible representation of $C^{*}(G(u), \mathscr{B})$. Then $\operatorname{Ind}_{G(u)}^{G} L$ is an irreducible representation of $C^{*}(G, \mathscr{B})$.

Our proof of Theorem 2.1 follows that of [6, Theorem 5] very closely. Because the notation for the convolution of sections, inner products and actions is virtually identical to the scalar-valued case, there are parts where the proof can be used mutatis mutandis from [6]. While this is one of the benefits of the Fell bundle formalism, Theorem 2.1 is a highly nontrivial generalization of the scalar version, and we have tried to be careful below to point out the places where we have had to adjust from working with scalar-valued functions to sections of a nontrivial Banach bundle. At the same time, it seemed prudent to retain enough of the original argument from the scalar case that the exposition remains readable.

2.1. Induced representations of Fell bundle $C^{*}$-algebras. We begin by recalling the construction of induced representations for Fell bundles over groupoids from [19, §4.1]. Let $q: \mathscr{B} \rightarrow G$ be a separable Fell bundle and assume that $H$ is a closed subgroupoid of $G$. Let $q_{H}:\left.\mathscr{B}\right|_{H} \rightarrow H$ be the Fell bundle obtained by restriction to $H$. Then $G_{H^{(0)}}=s^{-1}\left(H^{(0)}\right)$ is an $\left(H^{G}, H\right)$-equivalence, where $H^{G}$ is the imprimitivity groupoid $\left(G_{H^{(0)}} *_{s} G_{H^{(0)}}\right) / H$. If $\sigma: H^{G} \rightarrow G$ is the continuous map given by $\sigma([x, y])=x y^{-1}$, the pull-back Fell bundle $\sigma^{*} q: \sigma^{*} \mathscr{B} \rightarrow H^{G}$ is the Fell bundle $\sigma^{*} \mathscr{B}=\left\{([x, y], b):[x, y] \in H^{G}, b \in \mathscr{B}, \sigma([x, y])=q(b)\right\}$ with bundle map $\sigma^{*} q([x, y], b)=[x, y]$. Then $\mathscr{E}=q^{-1}\left(G_{H^{(0)}}\right)$ is a $\sigma^{*} \mathscr{B}-\left.\mathscr{B}\right|_{H^{-}}$-equivalence with

\footnotetext{
${ }^{1}$ The issue is that in the convolution algebra, the involution for groupoids has no modular function (since groupoids don't have modular functions until a quasi-invariant measure is picked). The modular function then reappears in the integrated forms of representations.
} 
the left action of $\sigma^{*} \mathscr{B}$ given by $([x, y], b) \cdot e=b e$ if $q(e)=y h$, the right action of $\left.\mathscr{B}\right|_{H}$ given by $e \cdot b=e b$, and the left and right inner products on $\mathscr{E} *_{s} \mathscr{E}$ given by

$$
{ }_{\sigma^{*} \mathscr{B}}\langle e, f\rangle=\left([q(e), q(f)], e f^{*}\right) \text { and }\langle e, f\rangle_{\left.\mathscr{B}\right|_{H}}=e^{*} f .
$$

Therefore $\Gamma_{c}\left(G_{H^{(0)}} ; \mathscr{E}\right)$ is a pre-imprimitivity bimodule with actions and inner products determined by

$$
\begin{aligned}
& F \cdot \varphi(z)=\int_{G} F([z, y]) \varphi(y) d \lambda_{s(z)}(y), \\
& \varphi \cdot g(z)=\int_{H} \varphi(z h) g\left(h^{-1}\right) d \alpha^{s(z)}(h), \\
& \langle\varphi, \psi\rangle_{\star}(h)=\int_{G} \varphi(y)^{*} \psi(y h) d \lambda_{r(h)}(y), \\
& { }_{\star}\langle\varphi, \psi\rangle([x, y])=\int_{H} \varphi(x h) \psi(y h)^{*} d \alpha^{s(x)}(h) .
\end{aligned}
$$

The completion $X=X_{H}^{G}$ is a $C^{*}\left(H^{G}, \sigma^{*} \mathscr{B}\right)-C^{*}\left(H,\left.\mathscr{B}\right|_{H}\right)$-imprimitivity bimodule.

If $L$ is a representation of $C^{*}\left(H,\left.\mathscr{B}\right|_{H}\right)$, then we write $\mathrm{X}$-Ind $L$ for the representation of $C^{*}\left(H^{G}, \sigma^{*} \mathscr{B}\right)$ induced via $X$. Recall (see, for example, 13. Proposition 2.66]) that $\mathrm{X}$-Ind $L$ acts on the completion $\mathcal{H}_{\text {Ind } L}$ of $X \odot \mathcal{H}_{L}$ with respect to

$$
(\varphi \otimes h \mid \psi \otimes k)=\left(L\left(\langle\psi, \varphi\rangle_{\star}\right) h \mid k\right)_{\mathcal{H}_{L}}
$$

via

$$
(\mathrm{X}-\text { Ind } L)(F)(\varphi \otimes h)=F \cdot \varphi \otimes h .
$$

The induced representation of $C^{*}(G ; \mathscr{B})$ acts on $\mathcal{H}_{\text {Ind } L}$ by

$$
\left(\operatorname{Ind}_{H}^{G} L\right)(f)(\varphi \otimes h)=f * \varphi \otimes h,
$$

where $f * \varphi(z)=\int_{G} f(y) \varphi\left(y^{-1} z\right) d \lambda^{r(z)}(y)$ for $f \in \Gamma_{c}(G, \mathscr{B})$ and $\varphi \in \Gamma_{c}\left(G_{H^{(0)}}, \mathscr{E}\right)$.

2.2. The proof of Theorem 2.1. For the proof, we consider the case $H=G(u)$ for some $u \in G^{(0)}$. Then $H^{(0)}=\{u\}$ and $G_{H^{(0)}}=G_{u}$.

Let $L$ be an irreducible representation of $C^{*}(G(u), \mathscr{B})$. Since $\mathrm{X}$ is a $C^{*}\left(G(u)^{G}, \sigma^{*} \mathscr{B}\right)-C^{*}\left(G(u),\left.\mathscr{B}\right|_{G(u)}\right)$-imprimitivity bimodule, [13, Corollary 3.32] implies that $\mathrm{X}$-Ind $L$ is an irreducible representation of $C^{*}\left(G(u)^{G}, \sigma^{*} \mathscr{B}\right)$. To prove the theorem, we just need to see that any $T$ in the commutant of $\operatorname{Ind}_{G(u)}^{G} L$ is a scalar multiple of the identity. It will suffice to see that any such $T$ commutes with $(\mathrm{X}-\mathrm{Ind} L)(F)$ for all $F \in \Gamma_{c}\left(G(u)^{G}, \sigma^{*} \mathscr{B}\right)$. Hence, given such an $F$, we need to produce a net $\left\{f_{i}\right\}$ in $\Gamma_{c}(G, \mathscr{B})$ such that

$$
\left(\operatorname{Ind}_{G(u)}^{G} L\right)\left(f_{i}\right) \rightarrow(\mathrm{X}-\operatorname{Ind} L)(F)
$$

in the weak operator topology. We will arrange that this net is uniformly bounded in the $\|\cdot\|_{I}$-norm on $\Gamma_{c}(G, \mathscr{B})$ - so that the net $\left\{\left(\operatorname{Ind}_{G(u)}^{G} L\right)\left(f_{i}\right)\right\}$ is uniformly bounded in $B\left(\mathcal{H}_{\text {Ind } L}\right)$. Then we just have to arrange that

$$
\left(\left(\operatorname{Ind}_{G(u)}^{G} L\right)\left(f_{i}\right)(\varphi \otimes h) \mid \psi \otimes k\right) \rightarrow((\mathrm{X}-\operatorname{Ind} L)(F)(\varphi \otimes h) \mid \psi \otimes k)
$$

for all $\varphi, \psi \in \Gamma_{c}\left(G_{u}, \mathscr{E}\right)$ and $h, k \in \mathcal{H}_{\text {Ind } L}$.

As in the proof of [6, Theorem 5], the following lemma is the essential ingredient in our proof. 
Lemma 2.2. Suppose that $F \in \Gamma_{c}\left(G(u)^{G}, \sigma^{*} \mathscr{B}\right)$. Then there is a compact set $C_{F}$ in $G$ such that for each compact set $K \subset G_{u}$ there is an $f_{K} \in \Gamma_{c}(G, \mathscr{B})$ such that

(a) $f_{K}\left(z y^{-1}\right)=F([z, y])$ for all $(z, y) \in K \times K$,

(b) $\operatorname{supp} f_{K} \subset C_{F}$ and

(c) $\left\|f_{K}\right\|_{I} \leq\|F\|_{I}+1$.

In order to prove Lemma 2.2, we need a version of [6, Lemma 7] for semicontinuous functions.

Lemma 2.3. Suppose that $f$ is a nonnegative upper semicontinuous function on $G$ with compact support and that $K \subset G$ is a compact set such that

$$
\int_{K} f(x) d \lambda^{u}(x) \leq M \quad \text { for all } u \in G^{(0)} .
$$

Then there is a neighborhood $V$ of $K$ such that

$$
\int_{V} f(x) d \lambda^{u}(x) \leq M+1 \quad \text { for all } u \in G^{(0)} .
$$

Proof. Let $K_{1}$ be a compact neighborhood of $K$ and $\left\{V_{n}\right\}$ a countable fundamental system of neighborhoods of $K$ in $K_{1}$ such that $V_{n+1} \subset V_{n}$. Assuming to the contrary that no $V$ as prescribed in the lemma exists, then we can find a sequence $\left\{u_{n}\right\} \subset G^{(0)}$ such that

$$
\int_{V_{n}} f(x) d \lambda^{u_{n}}(x)>M+1 .
$$

As in [6], we can assume that $u_{n} \rightarrow u_{0}$. The dominated convergence theorem implies that

$$
\int_{V_{n}} f(x) d \lambda^{u_{0}}(x) \rightarrow \int_{K} f(x) d \lambda^{u_{0}}(x) .
$$

In particular, there is an $n_{1}$ such that

$$
\int_{V_{n_{1}}} f(x) d \lambda^{u_{0}}(x) \leq M+\frac{1}{2} .
$$

Let $W_{1}$ be an open set such that $K \subset W_{1} \subset \bar{W}_{1} \subset V_{n_{1}}$. Then $1_{\bar{W}_{1}} f$ is upper semicontinuous and

$$
\int_{\bar{W}_{1}} f(x) d \lambda^{u_{0}} \leq M+\frac{1}{2} .
$$

Let $0<\varepsilon<\frac{1}{2}$. Using [10, Lemma 3.4] we can find $g \in C_{c}^{+}(G)$ such that $f(x) \leq g(x)$ for all $x \in \bar{W}_{1}$ and

$$
\int_{\bar{W}_{1}} f(x) d \lambda^{u_{0}}(x) \leq \int_{G} g(x) d \lambda^{u_{0}}(x)<M+\frac{1}{2}+\varepsilon .
$$

Let $W$ be open such that $K \subset W \subset \bar{W} \subset W_{1}$ and let $f_{0} \in C_{c}^{+}(G)$ be such that $\left.f_{0}\right|_{\bar{W}}=g, f_{0} \leq g$, and $\operatorname{supp} f_{0} \subset W_{1}$. Then

$$
\int_{G} f_{0}(x) d \lambda^{u_{0}}(x)<M+\frac{1}{2}+\varepsilon .
$$

However, since $\left\{\lambda^{u}\right\}$ is a Haar system,

$$
\int_{G} f_{0}(x) d \lambda^{u_{m}}(x) \rightarrow \int_{G} f_{0}(x) d \lambda^{u_{0}}(x) .
$$


Therefore, for large $n$, we have that $\int_{G} f_{0}(x) d \lambda^{u_{m}}(x)<M+1$. However, for large $n$, we have $V_{n} \subset W$ and therefore

$$
\begin{aligned}
\int_{G} f_{0}(x) d \lambda^{u_{n}}(x) & \geq \int_{V_{n}} f_{0}(x) d \lambda^{u_{n}}(x)=\int_{V_{n}} g(x) d \lambda^{u_{n}}(x) \\
& \geq \int_{V_{n}} f(x) d \lambda^{u_{n}}(x)>M+1 .
\end{aligned}
$$

This leads to a contradiction and the proof is complete.

Proof of Lemma 2.2. The map $(z, y) \mapsto z y^{-1}$ is continuous on $G_{u} \times G_{u}$ and factors through the orbit map $\pi: G_{u} \times G_{u} \rightarrow G(u)^{G}$. Moreover, the continuous map $\sigma: G(u)^{G} \rightarrow G$ defined via $\sigma([z, y])=z y^{-1}$ is injective. We let $C_{F}$ be a compact neighborhood of $\sigma(\operatorname{supp} F)$.

Fix a compact set $K \subset G_{u}$. Then the restriction of $\sigma$ to the compact set $\pi(K \times K)$ is a homeomorphism. Using the vector-valued Tietze Extension Theorem ([10, Proposition A.5]) we can find a section $\tilde{f}_{K} \in \Gamma_{c}(G, \mathscr{B})$ such that supp $\tilde{f}_{K} \subset C_{F}$ and such that $\tilde{f}_{K}\left(z y^{-1}\right)=F([z, y])$ for all $(z, y) \in K \times K$.

Let $K_{G}:=\sigma(\pi(K \times K)) \subset G$. If

$$
\int_{K_{G}}\left\|\tilde{f}_{K}(y)\right\| d \lambda^{w}(y) \neq 0
$$

then $K_{G} \cap G^{w} \neq \emptyset$. Therefore there is $z \in K$ such that $r(z)=w$. Then by left invariance

$$
\begin{aligned}
\int_{K_{G}}\left\|\tilde{f}_{K}(y)\right\| d \lambda^{w}(y) & =\int_{G} \mathbf{1}_{K_{G}}(z y)\left\|\tilde{f}_{K}(z y)\right\| d \lambda^{w}(y) \\
& =\int_{G} \mathbf{1}_{K_{G}}\left(z y^{-1}\right)\left\|\tilde{f}_{K}\left(z y^{-1}\right)\right\| d \lambda_{u}(y) \\
& =\int_{G} \mathbf{1}_{K_{G}}\left(z y^{-1}\right)\|F([z, y])\| d \lambda_{u}(y) \\
& \leq\|F\|_{I}
\end{aligned}
$$

Similarly, if

$$
\int_{K_{G}}\left\|\tilde{f}_{K}\left(y^{-1}\right)\right\| d \lambda^{w}(y) \neq 0
$$

then as before there is a $z \in K$ such that $r(z)=w$ and

$$
\begin{aligned}
\int_{K_{G}}\left\|\tilde{f}_{K}\left(y^{-1}\right)\right\| d \lambda^{w}(y) & =\int_{G} \mathbf{1}_{K_{G}}(z y)\left\|\tilde{f}_{K}\left(y^{-1} z^{-1}\right)\right\| d \lambda^{w}(y) \\
& =\int_{G} \mathbf{1}_{K_{G}}\left(z y^{-1}\right)\left\|\tilde{f}_{K}\left(y z^{-1}\right)\right\| d \lambda_{u}(y)
\end{aligned}
$$

which, since $K_{G}^{-1}=K_{G}$, is

$$
\begin{aligned}
& =\int_{G} \mathbf{1}_{K_{G}}\left(y z^{-1}\right)\left\|\tilde{f}_{K}\left(y z^{-1}\right)\right\| d \lambda_{u}(y) \\
& \leq \int_{G}\|F([y, z])\| d \lambda_{u}(y) \leq\|F\|_{I} .
\end{aligned}
$$


Using Lemma 2.3, we can find a neighborhood $V$ of $K_{G}$ contained in $C_{F}$ such that both

$$
\int_{V}\left\|\tilde{f}_{K}(y)\right\| d \lambda^{w}(y) \quad \text { and } \quad \int_{V}\left\|\tilde{f}_{K}\left(y^{-1}\right)\right\| d \lambda^{w}(y)
$$

are bounded by $\|F\|_{I}+1$ for all $w \in G^{(0)}$. Since $K_{G}$ is symmetric we can assume that $V=V^{-1}$ as well. Using the vector-valued Tietze Extension Theorem, we let $f_{K}$ be any element of $\Gamma_{c}(G, \mathscr{B})$ such that $f_{K}=\tilde{f}_{K}$ on $K_{G}$, supp $f_{K} \subset V$ and $\left\|f_{K}(x)\right\| \leq\left\|\tilde{f}_{K}(x)\right\|$ everywhere. Then $f_{K}$ satisfies the conclusion of the lemma.

Proof of Theorem 2.1. For each $K \subset G_{u}$, let $f_{K}$ be as in Lemma 2.3. Then $\left\{f_{K}\right\}$ and $\left\{\left(\operatorname{Ind}_{G(u)}^{G} L\right)\left(f_{K}\right)\right\}$ are nets indexed by increasing $K$. Notice that

$$
\begin{aligned}
& \left(\left(\operatorname{Ind}_{G(u)}^{G} L\right)\left(f_{K}\right)\left(\varphi \otimes_{G(u)} h\right) \mid \psi \otimes_{G(u)} k\right) \\
& \quad-\left((\mathrm{X}-\operatorname{Ind} L)(F)\left(\varphi \otimes_{G(u)} h\right) \mid \psi \otimes_{G(u)} k\right) \\
& \quad=\left(L\left(\left\langle\psi, f_{K} * \varphi-F \cdot \varphi\right\rangle_{\star}\right) h \mid k\right) .
\end{aligned}
$$

Furthermore, using the invariance of the Haar system on $G$, we can compute as follows:

$$
\begin{aligned}
\left\langle\psi, f_{K} * \varphi\right\rangle_{\star}(s) & =\int_{G} \psi(x)^{*} f_{K} * \varphi(x s) d \lambda_{u}(x) \\
& =\int_{G} \int_{G} \psi(x)^{*} f_{K}\left(x z^{-1}\right) \varphi(z s) d \lambda_{u}(z) d \lambda_{u}(x),
\end{aligned}
$$

while on the other hand,

$$
\begin{aligned}
\langle\psi, F \cdot \varphi\rangle_{\star}(s) & =\int_{G} \psi(x)^{*} F \cdot \varphi(x s) d \lambda_{u}(x) \\
& =\int_{G} \int_{G} \psi(x)^{*} F([x s, z]) \varphi(z) d \lambda_{u}(z) d \lambda_{u}(x) \\
& =\int_{G} \int_{G} \psi(x)^{*} F\left(\left[x, z s^{-1}\right]\right) \varphi(z) d \lambda_{u}(z) d \lambda_{u}(x) \\
& =\int_{G} \int_{G} \psi(x)^{*} F([x, z]) \varphi(z s) d \lambda_{u}(z) d \lambda_{u}(x) .
\end{aligned}
$$
have

Notice that $\operatorname{supp}\langle\psi, \varphi\rangle_{\star} \subset(\operatorname{supp} \psi)(\operatorname{supp} \varphi)$. Since $\operatorname{supp} f_{K} \subset C_{F}$ for all $K$, we

$$
\operatorname{supp} f_{K} * \varphi \subset\left(\operatorname{supp} f_{K}\right)(\operatorname{supp} \varphi) \subset C_{F}(\operatorname{supp} \varphi) \text {. }
$$

Therefore if (2.2) does not vanish, then we must have $s \in(\operatorname{supp} \psi) C_{F}(\operatorname{supp} \varphi)$. Therefore there is a compact set $K_{0}$ - which does not depend on $K$ - such that both (2.2) and (2.3) vanish if $s \notin K_{0}$. Thus if $s \in K_{0}$ and if $K \supset(\operatorname{supp} \psi) \cup$ $(\operatorname{supp} \varphi) K_{0}^{-1}$, then the integrand in (2.2) and (2.3) are both zero or we must have $(x, z) \in K \times K$. Therefore we can replace $f_{K}\left(x z^{-1}\right)$ by $F([x, z])$, and then $f_{K} * \varphi-$ $F \cdot \varphi$ is the zero section whenever $K$ contains $(\operatorname{supp} \psi) \cup(\operatorname{supp} \varphi) K_{0}^{-1}$. Therefore the left-hand side of (2.1) is eventually zero, and the theorem follows.

The proof of the following theorem is similar to that of [6, Theorem 4] and we will omit it. The result will be useful in Section 4 . 
Theorem 2.4 (Induction in stages). Suppose that $q: \mathscr{B} \rightarrow G$ is a separable Fell bundle over a second countable locally compact Hausdorff groupoid $G$ and that $H$ and $K$ are closed subgroupoids of $G$ with $H \subset K$. Assume that $H, K$, and $G$ have Haar systems. If $L$ is a representation of $C^{*}(H, \mathscr{B})$, then

$$
\operatorname{Ind}_{H}^{G} L \quad \text { and } \quad \operatorname{Ind}_{K}^{G}\left(\operatorname{Ind}_{H}^{K} L\right)
$$

are equivalent representations of $C^{*}(G, \mathscr{B})$.

\section{The Strong-Effros-Hahn Induction Property for Fell Bundles}

Recall that, for a Fell bundle $q: \mathscr{B} \rightarrow G$ over a groupoid $G, A=\Gamma_{0}\left(G^{(0)} ; \mathscr{B}\right)$ is a $C^{*}$-algebra that we call the $C^{*}$-algebra of $\mathscr{B}$ over $G^{(0)}$. Then $A$ is a $C_{0}\left(G^{(0)}\right)$ algebra and we let $\sigma_{A}: \operatorname{Prim} A \rightarrow G^{(0)}$ be the associated structure map (see, for example, [20, §C.1]). If $u \in G^{(0)}$, let $p_{u}: A \rightarrow A(u)$ be the quotient map with kernel $I(u)$. Note that if $P \in \operatorname{Prim} A$, then $u=\sigma_{A}(P)$ is the unique $u \in G^{(0)}$ such that $P \supset I(u)$. Moreover, $\operatorname{Prim} A$ is naturally identified with the disjoint union of the Prim $A(u)$ [20, Proposition C.5].

Recall that $G$ acts on Prim $A$ via the Rieffel correspondence

$$
h_{x}: \operatorname{Prim} A(s(x)) \rightarrow \operatorname{Prim} A(r(x))
$$

(see [7. §2]). Thus, if $P \in \operatorname{Prim} A$ and if $x \in G$ is such that $\sigma_{A}(P)=s(x)$, then $x \cdot P=h_{x}(P)$. Since the $G$-action $h_{x}$ also maps $I(s(x))$ to $I(r(x))$ (see [13. Proposition 3.24]), the stability group $G_{P}$ of $P$ is a subgroup of the stability group $G(u)$, where $u=\sigma_{A}(P)$.

Suppose now that $q: \mathscr{B} \rightarrow G$ is a separable Fell bundle over a group $G 2$ If $L: C^{*}(G, \mathscr{B}) \rightarrow B(\mathcal{W})$ is a representation, then there is a strictly continuous, nondegenerate $*$-homomorphism $\pi: \mathscr{B} \rightarrow B(\mathcal{W})$ such that

$$
L(f)=\int_{G} \pi(f(s)) \Delta_{G}(s)^{-\frac{1}{2}} d \mu_{G}(s) .
$$

This is a consequence of [8, Lemma 1.3]. (The appearance of the modular function in (3.1) is a consequence of our convention of treating $G$ as a groupoid: see 8 , Remark 1.5].) Notice that $\left.\pi\right|_{A}$ is a representation of $A$ on $\mathcal{W}$. Let $I$ be an ideal in $A=B(e)$. Then we say that $L$ or $\pi$ has kernel $I$ if $\operatorname{ker}\left(\left.\pi\right|_{A}\right)=I$.

In analogy with [2, Definition 1.1], we make the following definition.

Definition 3.1. We say that a Fell bundle $q: \mathscr{B} \rightarrow G$ over a groupoid $G$ with $C^{*}$-algebra $A$ over $G^{(0)}$ satisfies the strong-Effros-Hahn Induction Property (strongEHI) if given $P \in \operatorname{Prim} A$ and an irreducible representation $L$ of $C^{*}\left(G_{P}, \mathscr{B}\right)$ with kernel $P$, then $\operatorname{Ind}_{G_{P}}^{G} L$ is irreducible.

\section{The STRONG-EHI FOR HOMOGENEOUS REPRESENTATIONS}

In this section we prove our main result, which asserts that the strong-EHI property holds under the additional assumption that the restriction of the representation to $A$ is homogeneous.

\footnotetext{
${ }^{2}$ Notice that in this case the underlying Banach bundle is continuous [1 Lemma 3.30].
} 
Theorem 4.1. Let $q: \mathscr{B} \rightarrow G$ be a saturated, separable Fell bundle over a locally compact groupoid $G$ and suppose that $P \in \operatorname{Prim} A$ where $A=\Gamma_{0}\left(G^{(0)} ; \mathscr{B}\right)$ is the associated $C^{*}$-algebra over $G^{(0)}$. Suppose that $L$ is an irreducible representation of $C^{*}\left(G_{P},\left.\mathscr{B}\right|_{G_{P}}\right)$ which is the integrated form of $\pi:\left.\mathscr{B}\right|_{G_{P}} \rightarrow B(\mathcal{W})$ with $\left.\pi\right|_{A}$ homogeneous with kernel $P$. Then $\operatorname{Ind}_{G_{P}}^{G} L$ is an irreducible representation of $C^{*}(G, \mathscr{B})$.

Remark 4.2. Let $L$ be a representation of $C^{*}\left(G_{P}, \mathscr{B}\right)$ with kernel $P$. Then Theorem 2.4 implies that $\operatorname{Ind}_{G_{P}}^{G} L$ and $\operatorname{Ind}_{G(u)}^{G}\left(\operatorname{Ind}_{G_{P}}^{G(u)} L\right)$ are equivalent representations, where $u=\sigma_{A}(P)$. Together with Theorem 2.1 this shows that the irreducibility of $\operatorname{Ind}_{G_{P}}^{G(u)} L$ implies that of $\operatorname{Ind}_{G_{P}}^{G} L$. Hence, in order to show that a Fell bundle satisfies strong-EHI, it will suffice to consider the case where $G$ is a group.

In view of Remark 4.2, we will assume for the remainder of this section that $G$ is a group. Hence, we fix a Fell bundle $q: \mathscr{B} \rightarrow G$ over a group $G$ and let $A=B(e)$ be the corresponding $C^{*}$-algebra.

To start, let $H$ be any closed subgroup of $G$. Fix Haar measures $\mu$ and $\nu$ on $G$ and $H$, respectively. Let $\rho: G \rightarrow(0, \infty)$ be a continuous function satisfying

$$
\rho(x h)=\frac{\Delta_{H}(h)}{\Delta_{G}(h)} \rho(x) \quad \text { for all } x \in G \text { and } h \in H .
$$

For convenience later, we normalize $\rho$ so that $\rho(e)=1$. Then there is a quasiinvariant measure $\bar{\mu}$ on $G / H$ (see, for example, [13, Lemma C.2]) such that

$$
\begin{aligned}
\int_{G} f(x) \rho(x) d \mu(x) \\
\quad=\int_{G / H} \int_{H} f(x h) d \nu(h) d \bar{\mu}(\dot{x}) \quad \text { for all } f \in C_{c}(G) .
\end{aligned}
$$

In fact, $\bar{\mu}$ is quasi-invariant when viewed as a measure on the unit space of the transformation groupoid $G \times G / H$ (which we identify with $G / H$ ). Recall that two elements $(x, y H)$ and $(z, w H)$ in $G \times G / H$ are composable provided that $w H=$ $x^{-1} y H$ and $(x, y H)\left(z, x^{-1} y H\right)=(x z, y H)$. The inverse of $(x, y H)$ is $\left(x^{-1}, x^{-1} y H\right)$. It follows that $s(x, y H)=x^{-1} y H$ and $r(x, y H)=y H$. The Haar system on the transformation groupoid is given by $\lambda=\left\{\mu \times \epsilon_{y H}\right\}_{y H \in G / H}$.

Lemma 4.3. The modular function on the transformation groupoid $G \times G / H$ with respect to the quasi-invariant measure $\bar{\mu}$ defined in (4.2) is

$$
\delta(x, y H)=\Delta_{G}(x) \frac{\rho(y)}{\rho\left(x^{-1} y\right)} .
$$

Proof. Let $F \in C_{c}(G \times G / H)$. Then

$$
\begin{aligned}
\lambda(F) & =\int_{G / H} \int_{G} F(x, y H) d \mu(x) d \bar{\mu}(\dot{y}) \\
& =\int_{G / H} \int_{G} F\left(x^{-1}, y H\right) \Delta_{G}\left(x^{-1}\right) d \mu(x) d \bar{\mu}(\dot{y})
\end{aligned}
$$


which, using Fubini and [13, Lemma C.2], is

$$
\begin{gathered}
=\int_{G / H} \int_{G / H} \int_{H} F\left((x h)^{-1},(x h)^{-1} y\right) \Delta_{G}\left((x h)^{-1}\right) \\
\frac{\rho\left((x h)^{-1} y\right)}{\rho(y)} \frac{1}{\rho(x h)} d \nu(h) d \bar{\mu}(\dot{y}) d \bar{\mu}(\dot{x}) \\
=\int_{G / H} \int_{G} F\left(x^{-1}, x^{-1} y H\right) \Delta_{G}\left(x^{-1}\right) \frac{\rho\left(x^{-1} y\right)}{\rho(y)} d \mu(x) d \bar{\mu}(\dot{y}) .
\end{gathered}
$$

It follows that

$$
\delta\left((x, y H)^{-1}\right)=\delta\left(x^{-1}, x^{-1} y H\right)=\Delta_{G}\left(x^{-1}\right) \frac{\rho\left(x^{-1} y\right)}{\rho(y)} .
$$

The conclusion follows.

We now fix a representation $L$ of $C^{*}(H, \mathscr{B})$ on $\mathcal{W}$, and assume that it is the integrated form of $\pi:\left.\mathscr{B}\right|_{H} \rightarrow B(\mathcal{W})$ as in (3.1). Note that the map $[x, y] \mapsto$ $\left(x y^{-1}, x H\right)$ allows us to identify the imprimitivity groupoid $H^{G}=(G \times G) / H$ with the transformation groupoid $G \times G / H$. Then we can complete $\Gamma_{c}(G ; \mathscr{B})$ to an imprimitivity bimodule $\mathrm{X}$ between $C^{*}\left(G \times G / H, \mathrm{pr}_{1}^{*} \mathscr{B}\right)$ and $C^{*}(H, \mathscr{B})$, where $\operatorname{pr}_{1}: G \times G / H$ is the projection onto the $G$-factor. Hence $\operatorname{pr}_{1}^{*} \mathscr{B}=\{(y, x H, b)$ : $b \in B(y)\}$. Then the induced representations $\mathrm{X}$-Ind $L$ of $C^{*}\left(G \times G / H, \mathrm{pr}_{1}^{*} \mathscr{B}\right)$ and $\operatorname{Ind}_{H}^{G} L$ of $C^{*}(G, \mathscr{B})$ both act on the completion of

$$
\Gamma_{c}(G ; \mathscr{B}) \odot \mathcal{W}
$$

with respect to the pre-inner product

$$
\begin{aligned}
(f \otimes \xi \mid g \otimes \eta)=\left(L\left(\langle g, f\rangle_{\star}\right) \xi \mid \eta\right) & \\
= & \int_{H}\left(\pi\left(\langle g, f\rangle_{\star}(h)\right) \xi \mid \eta\right) \Delta_{H}(h)^{-\frac{1}{2}} d \nu(h) \\
& =\int_{H} \int_{G}\left(\pi\left(g\left(x^{-1}\right)^{*} f\left(x^{-1} h\right)\right) \xi \mid \eta\right) \Delta_{H}(h)^{-\frac{1}{2}} d \mu(x) d \nu(h) \\
& =\int_{H} \int_{G}\left(\pi\left(g(x)^{*} f(x h)\right) \xi \mid \eta\right) \Delta_{G}(x)^{-1} \Delta_{H}(h)^{-\frac{1}{2}} d \mu(x) d \nu(h) \\
& =\int_{H} \int_{G / H} \int_{H}\left(\pi\left(g(x r)^{*} f(x r h)\right) \xi \mid \eta\right) \Delta_{G}(x r)^{-1} \Delta_{H}(h)^{-\frac{1}{2}} \rho(x r)^{-1} \\
& =\int_{G / H}(f \otimes \xi \mid g \otimes \eta)_{x H} d \bar{\mu}(\dot{x}),
\end{aligned}
$$

where we define

$$
\begin{aligned}
& (f \otimes \xi \mid g \otimes \eta)_{x H} \\
& \quad=\int_{H} \int_{H}\left(\pi\left(g(x r)^{*} f(x h)\right) \xi \mid \eta\right) \Delta_{G}(x r)^{-1} \Delta_{H}\left(r^{-1} h\right)^{-\frac{1}{2}} \rho(x r)^{-1} d \nu(h) d \nu(r) .
\end{aligned}
$$

As we described in Section 2.1, the induced representations are given by

$(\mathrm{X}-\mathrm{Ind} L)(F)(g \otimes \eta)=F \cdot g \otimes \eta \quad$ and $\quad\left(\operatorname{Ind}_{H}^{G} L\right)(f)(g \otimes \eta)=f * g \otimes \eta$, 
where

$$
F \cdot g(x)=\int_{G} F(y, x H) g\left(y^{-1} x\right) d \mu(y) \quad \text { and } \quad f * g(x)=\int_{G} f(y) g\left(y^{-1} x\right) d \mu(y) .
$$

We will need the following technical lemma.

Lemma 4.4. For each $x \in G$, the sesquilinear form $(\cdot \mid \cdot)_{x H}$ is positive semidefinite on $\Gamma_{c}(G ; \mathscr{B}) \odot \mathcal{W}$ and therefore a pre-inner product.

Proof. It is not hard to check that $f \mapsto(f \otimes \xi \mid f \otimes \xi)$ is continuous in the inductive limit topology. It follows from [11, Lemma 6.1] that if $\left\{a_{\kappa}\right\}$ is an approximate unit for $A$ and $f \in \Gamma_{c}(G ; \mathscr{B})$, then $a_{\kappa} \cdot f \rightarrow f$ in the inductive limit topology. Hence

$$
\left(\sum_{i=1}^{n} f_{i} \otimes \xi_{i} \mid \sum_{j=1}^{n} f_{j} \otimes \xi_{j}\right)=\lim _{\kappa}\left(\sum_{i=1}^{n} a_{\kappa} \cdot f_{i} \otimes \xi_{i} \mid \sum_{j=1}^{n} a_{\kappa} \cdot f_{j} \otimes \xi_{j}\right) .
$$

Since $B(x)$ is an $A-A$-imprimitivity bimodule, sums of the form $\sum_{r} c_{r} c_{r}^{*}$, with each $c_{r} \in B(x)$, are dense in $A^{+}$(see [11, Lemma 6.3]). Since each $a_{\kappa} \geq 0$ in $A$, this implies that we can replace $a_{\kappa}$ by a sum $\sum_{r} c_{r}^{\kappa}\left(c_{r}^{\kappa}\right)^{*}$ with $c_{r}^{\kappa} \in B(x)$ and still have (4.6) hold.

But then since $\rho(e)=1$ we have

$$
\Delta_{G}(x r)^{-1} \rho(x r)^{-1} \Delta_{H}\left(r^{-1} h\right)^{-\frac{1}{2}}=\Delta_{G}(x)^{-1} \Delta_{H}(h r)^{-\frac{1}{2}} \rho(x)^{-1}
$$

and

$$
\left(\sum_{i} a_{\kappa} \cdot f_{i} \otimes \xi_{i} \mid \sum_{j} a_{\kappa} \cdot f_{j} \otimes \xi_{j}\right)_{x H}=\sum_{r}\left(\eta_{r}^{\kappa} \mid \eta_{r}^{\kappa}\right)
$$

where

$$
\eta_{r}^{\kappa}=\sum_{i} \int_{H} \Delta_{G}(x)^{-\frac{1}{2}} \Delta_{H}(h)^{-\frac{1}{2}} \pi\left(\left(c_{r}^{\kappa}\right)^{*} f_{i}(x h)\right) \xi_{i} d \nu(h) .
$$

Since $\sum_{k}\left(\eta_{k} \mid \eta_{k}\right)$ is nonnegative, it follows from (4.6) that our form is a pre-inner product as claimed.

In view of Lemma 4.4 the (Hausdorff) completion of $\Gamma_{c}(G ; \mathscr{B}) \odot \mathcal{W}$ with respect to $(\cdot \mid \cdot)_{x H}$ is a Hilbert space $\mathcal{V}(x H)$. We denote the image of $f \otimes \xi$ in $\mathcal{V}(x H)$ by $f \otimes_{x H} \xi$. Note that the class of $f \otimes_{x H} \xi$ depends only on $\left.f\right|_{x H}$. Furthermore, in view of the Tietze Extension Theorem for upper semicontinuous Banach bundles [10, Proposition A.5], every $f_{0} \in \Gamma_{c}(x H ; \mathscr{B})$ is the restriction of some $f \in$ $\Gamma_{c}(G ; \mathscr{B})$. Using [20, Proposition F.8], we can form a Borel Hilbert bundle $G / H * \mathscr{V}$, and identify the space of both induced representations with the direct integral $L^{2}(G / H * \mathscr{V}, \bar{\mu})$. We will write $f \otimes \xi$ for the element in $L^{2}(G / H * \mathscr{V}, \bar{\mu})$ given by $f \otimes \xi(x H)=f \otimes_{x H} \xi$.

If $b \in B(y) \subset \mathscr{B}$, then we define an operator $\iota_{\mathscr{B}}(b)$ on the vector space $\Gamma_{c}(G ; \mathscr{B})$ by

$$
\iota_{\mathscr{B}}(b) f(x)=\Delta_{G}(y)^{\frac{1}{2}} b f\left(y^{-1} x\right) .
$$

By [8, Lemma 1.2 and Remark 1.5], $\iota_{\mathscr{B}}$ extends to a homomorphism, also denoted $\iota_{\mathscr{B}}$, of $\mathscr{B}$ into $M\left(C^{*}(G, \mathscr{B})\right)$. It follows that $\operatorname{Ind}_{H}^{G} L$ is the integrated form of $\hat{\Pi}: \mathscr{B} \rightarrow B\left(L^{2}(G / H * \mathscr{V}, \bar{\mu})\right)$ given by

$$
\hat{\Pi}(b)(f \otimes \xi)=\iota \mathscr{B}(b) f \otimes \xi .
$$


Note that $\hat{\Pi}$ is not decomposible; that is, it does not operate fibrewise on $L^{2}(G / H * \mathscr{V}, \bar{\mu})$. Nevertheless, it does play nice with the fibres and is related to a Borel $*$-functor for $\operatorname{pr}_{1} \mathscr{B}$ (see [10, Definition 4.5]).

Lemma 4.5. If $b \in B(y) \subset \mathscr{B}$, then we get an operator $\hat{\pi}(y, x H, b) \in$ $B\left(\mathcal{V}\left(y^{-1} x H\right), \mathcal{V}(x H)\right)$ of norm at most $\|b\|$ given by

$$
\begin{aligned}
\hat{\pi}(y, x H, b)\left(f \otimes_{y^{-1} x H} \xi\right)=\hat{\pi}_{0}(y, x H, b)(f) \otimes_{x H} \xi, \quad \text { where } \\
\hat{\pi}_{0}(y, x H, b)(f)(x h)=\delta(y, x H)^{\frac{1}{2}} b f\left(y^{-1} x h\right) .
\end{aligned}
$$

(Recall that $\delta$ is the modular function on $G \times G / H$ for the quasi-invariant measure $\bar{\mu}$ - see Lemma 4.3.)

Proof. Using $\delta(y, x H)=\Delta_{G}(y) \frac{\rho(x)}{\rho\left(y^{-1} x\right)}$ as well as the observation that $\frac{\rho(x)}{\rho\left(y^{-1} x\right)}=$ $\frac{\rho(x h)}{\rho\left(y^{-1} x h\right)}$ for any $h \in H$, we first check that

$$
(\hat{\pi}(y, x H, b)(f \otimes \xi) \mid g \otimes \eta)_{x H}=\left(f \otimes \xi \mid \hat{\pi}\left(y^{-1}, y^{-1} x H, b^{*}\right)(g \otimes \eta)\right)_{y^{-1} x H} .
$$

However, in $\tilde{A}$, we have $\|b\|^{2} 1_{\tilde{A}}-b^{*} b \geq 0$. Hence there is a $c \in \tilde{A}$ such that $\|b\|^{2} 1_{\tilde{A}}-b^{*} b=c^{*} c$. Thus, extending $\hat{\pi}(e, x H, a)$ to all $a \in \tilde{A}$ in the usual way, we have

$$
\begin{aligned}
\|b\|^{2} \| f & \otimes \xi\left\|_{y^{-1} x H}^{2}-\right\| \hat{\pi}(y, x H, b)(f \otimes \xi) \|_{x H}^{2} \\
& =\|b\|^{2}(f \otimes \xi \mid f \otimes \xi)_{y^{-1} x H}-(\hat{\pi}(y, x H, b)(f \otimes \xi) \mid \hat{\pi}(y, x H, b)(f \otimes \xi))_{x H} \\
& =\|b\|^{2}(f \otimes \xi \mid f \otimes \xi)_{y^{-1} x H}-\left(f \otimes \xi \mid \hat{\pi}\left(e, y^{-1} x H, b^{*} b\right)\right)(f \otimes \xi)_{y^{-1} x H} \\
& =\left(\hat{\pi}\left(e, y^{-1} x H, c\right)(f \otimes \xi) \mid \hat{\pi}\left(e, y^{-1} x H, c\right)(f \otimes \xi)\right)_{y^{-1} x H} \\
& \geq 0 .
\end{aligned}
$$

Thus $\hat{\pi}(y, x H, b)$ is a well-defined operator from $\mathcal{V}\left(y^{-1} x H\right)$ to $\mathcal{V}(x H)$ for any $x$, and has norm at most $\|b\|$.

Now it is not hard to see that $\hat{\pi}$ is a Borel $*$-functor on $\operatorname{pr}_{1} \mathscr{B}$, and more significantly, X-Ind $L$ is the integrated form of $\hat{\pi}$ - see [10, Proposition 4.10].

At this point, we want to fix a primitive ideal $P \in \operatorname{Prim} A$ and replace $H$ by $G_{P}$, where $G_{P}$ is the stability group for the $G$ action on Prim $A$ induced using the Rieffel correspondence: see [7, §2] and Section 3 for details. In particular, if $x \in G$, then

$$
B(x) \cdot P=(x \cdot P) \cdot B(x) .
$$

We let $\rho_{x G_{P}}$ be the representation of $A$ in $\mathcal{V}\left(x G_{P}\right)$ given by $a \mapsto \hat{\pi}\left(e, x G_{P}, a\right)$. While a priori the $\rho_{x G_{P}}$ do not seem related to $\left.\pi\right|_{A}$, they in fact are intimately so.

Lemma 4.6. The representation $\rho_{e G_{P}}$ defined above is equivalent to $\left.\pi\right|_{A}$.

Proof. We claim that the map $U: \mathcal{V}\left(G_{P}\right) \rightarrow \mathcal{W}$ defined via

$$
U\left(f \otimes_{e G_{P}} \xi\right)=\int_{e G_{P}} \Delta_{H}(h)^{-\frac{1}{2}} \pi(f(h)) \xi d \nu(h)
$$

is a unitary that intertwines $\rho_{e G_{P}}$ with $\left.\pi\right|_{A}$. 
Let $f \otimes \xi$ and $g \otimes \eta$ be two elements in $\Gamma_{c}(G, \mathscr{B}) \odot \mathcal{W}$. Then

$$
\begin{aligned}
& \left(U\left(f \otimes_{e G_{P}} \xi\right) \mid U\left(g \otimes_{e G_{P}} \eta\right)\right) \\
& =\left(\int_{G_{P}} \Delta_{H}(h)^{-\frac{1}{2}} \pi(f(h)) \xi d \nu(h) \mid \int_{G_{P}} \Delta_{H}(r)^{-\frac{1}{2}} \pi(g(r)) \eta d \nu(r)\right) \\
& =\int_{G_{P}} \int_{G_{P}}(\pi(f(h)) \xi \mid \pi(g(r)) \eta) \Delta_{H}(h)^{-\frac{1}{2}} \Delta_{H}(r)^{-\frac{1}{2}} d \nu(h) d \nu(r)
\end{aligned}
$$

which, using the fact that $\left.\pi\right|_{\mathscr{B}}$ is a $*$-homomorphism and equation (44), equals

$$
\begin{aligned}
& =\int_{G_{P}} \int_{G_{P}}\left(\pi\left(g(r)^{*} f(h)\right) \xi \mid \eta\right) \Delta_{G}(r)^{-1} \rho(r)^{-1} \Delta_{H}\left(r^{-1} h\right) d \nu(h) d \nu(r) \\
& =\left(f \otimes_{e G_{P}} \xi \mid g \otimes_{e G_{P}} \eta\right)_{e G_{P}} .
\end{aligned}
$$

The surjectivity of $U$ follows from the fact that there are enough sections and the fact that $\pi$ is nondegenerate (see [18, Section 3]). Thus $U$ is indeed a unitary that clearly intertwines $\rho_{e G_{P}}$ with $\left.\pi\right|_{A}$.

Since each $B(x)$ is an $A-A$-imprimitivity bimodule, we can form the induced representation $B(x)-\operatorname{Ind}\left(\rho_{e G_{P}}\right)$ of $A$ on $B(x) \otimes_{A} \mathcal{V}\left(e G_{P}\right)$.

Lemma 4.7. For each $x \in G, B(x)-\operatorname{Ind}\left(\rho_{e G_{P}}\right)$ is equivalent to $\rho_{x G_{P}}$.

Proof. Define $U: B(x) \odot \mathcal{V}\left(e G_{P}\right) \rightarrow \mathcal{V}\left(x G_{P}\right)$ by

$$
U\left(c \otimes f \otimes_{e G_{P}} \xi\right)=\Delta_{G}(x)^{\frac{1}{2}} \rho(x)^{\frac{1}{2}}\left(f_{c} \otimes_{x G_{P}} \xi\right),
$$

where $f_{c}$ is any section in $\Gamma_{c}(G ; \mathscr{B})$ satisfying

$$
f_{c}(x h)=c f(h) .
$$

To check that $U$ is isometric one can proceed as follows:

$$
\begin{aligned}
(U(c \otimes & \left.\left.f \otimes_{e G_{P}} \xi\right) \mid U\left(b \otimes g \otimes_{e G_{P}} \eta\right)\right)_{x G_{P}}=\Delta_{G}(x) \rho(x)\left(f_{c} \otimes_{x G_{P}} \xi \mid g_{b} \otimes_{x G_{P}} \eta\right)_{x G_{P}} \\
& =\int_{G_{P}} \int_{G_{P}}\left(\pi\left(g_{b}(x r)^{*} f_{c}(x h)\right) \xi \mid \eta\right) \Delta_{G}(x) \Delta_{G}(x r)^{-1} \Delta_{H}\left(r^{-1} h\right)^{-\frac{1}{2}} \\
& =\int_{G_{P}} \int_{G_{P}}\left(\pi\left(g(r)^{*} b^{*} c f(h)\right) \xi \mid \eta\right) \Delta_{G}(r)^{-1} \Delta_{H}\left(r^{-1} h\right)^{-\frac{1}{2}} \rho(r)^{-1} d \nu(h) d \nu(r) \\
& =\int_{G_{P}} \int_{G_{P}}\left(\pi\left(g(r)^{*} \hat{\pi}_{0}\left(e, e G_{P}, b^{*} c\right)(f)(h)\right) \xi \mid \eta\right) \Delta_{G}(r)^{-1} \Delta_{H}\left(r^{-1} h\right)^{-\frac{1}{2}} \\
& =\left(\rho_{e G_{P}}\left(b^{*} c\right)\left(f \otimes_{e} G_{P} \xi\right) \mid g \otimes_{e} G_{P} \eta\right)_{e G_{P}} \\
= & \left(c \otimes f \otimes_{e} G_{P} \xi \mid b \otimes g \otimes_{e} G_{P} \eta\right) .
\end{aligned}
$$

Clearly $U$ intertwines $B(x)-\operatorname{Ind}\left(\rho_{\left.e G_{P}\right)}\right)$ with $\rho_{x G_{P}}$.

Notice that $\left\{c \otimes f(h): f \in \Gamma_{c}(G, \mathscr{B}), c \in B(x)\right\}$ is dense in $B(x) \otimes_{A} B(h) \simeq$ $B(x h)$. Therefore the set of $f_{c}(x h)$ where $f_{c}(x h)=c f(h), f \in \Gamma_{c}(G, \mathscr{B})$, and $c \in B(x)$ is dense in $B(x h)$. [10, Lemma A.4] implies that $U$ has dense range.

Of course, just as with group dynamics (see [2, §2.3]), the homogeneity of $\left.\pi\right|_{A}$ will play a crucial role. As in [2, Remark 1.5], we will use [16, Lemme 1.5] or [3. Theorem 1.4] to characterize homogeneous representations $\rho$ of $A$ as those that 
have the property that for any ideal $I$ in $A$ such that $I \not \subset \operatorname{ker} \rho, \overline{\rho(I) \mathcal{H}_{\rho}}=\mathcal{H}_{\rho}$. Alternatively, note that $\rho$ is homogeneous if given any approximate identity $\left\{u_{i}\right\}$ for an ideal $I \not \subset \operatorname{ker} \rho$, we have $\rho\left(u_{i}\right) \rightarrow I_{\mathcal{H}_{\rho}}$ in the strong operator topology.

We need the following general Morita Equivalence result which we have not seen elsewhere $3^{3}$

Proposition 4.8. Let $A$ and $B$ be $C^{*}$-algebras and let $\mathrm{X}$ be an $A$-B-imprimitivity bimodule. Then $\pi$ is a homogeneous representation of $B$ if and only if $\mathrm{X}$-Ind $\pi$ is a homogeneous representation of $A$.

Proof. By symmetry, it suffices to show that $\pi$ homogeneous implies that X-Ind $\pi$ is homogeneous. So, we suppose that $\pi$ is homogeneous and that $K$ is an ideal in $A$ with $K \not \subset \operatorname{ker}(\mathrm{X}-\mathrm{Ind} \pi)$. Let $\left\{u_{i}\right\}$ be an approximate unit for $K$. As remarked above, it will suffice to see that $(\mathrm{X}$-Ind $\pi)\left(u_{i}\right) \rightarrow I$ in the strong operator topology. By the Rieffel correspondence $([13, \S 3.3])$, we can assume that $K=\mathrm{X}$-Ind $J$ for an ideal $J$ in $B$ with $J \not \subset \operatorname{ker} \pi$. Since $\pi$ is homogeneous, $\overline{\pi(J) \mathcal{H}_{\pi}}=\mathcal{H}_{\pi}$. Hence it will suffice to show that

$$
u_{i} \cdot x \otimes \pi(b) h \rightarrow x \otimes \pi(b) h
$$

in the Hilbert space $\mathbf{X} \otimes_{B} \mathcal{H}_{\pi}$ for all $x \in \mathbf{X}, b \in J$ and $h \in \mathcal{H}_{\pi}$.

However, $\mathrm{X} \cdot J$ is a $K-J$-imprimitivity bimodule (see [13, Proposition 3.25]). Hence for any $y \in \mathbf{X} \cdot J$, we have $u_{i} \cdot y \rightarrow y$ in $\mathbf{X}$ (for example, see equation (2.5) in the proof of [13, Corollary 2.7]). So in $\mathrm{X} \otimes_{B} \mathcal{H}_{\pi}$, we have

$$
\begin{aligned}
\left(u_{i} \cdot x \otimes \pi(b) h \mid u_{i} \cdot x \otimes \pi(b) h\right) & =\left(\pi\left(\left\langle u_{i} \cdot x, u_{i} \cdot x\right\rangle_{\star}\right) \pi(b) h \mid \pi(b) h\right) \\
& =\left(\pi\left(\left\langle u_{i} \cdot x \cdot b, u_{i} \cdot x \cdot b\right\rangle_{\star}\right) h \mid h\right),
\end{aligned}
$$

which converges to

$$
\left(\pi\left(\langle x, x\rangle_{\star}\right) \pi(b) h \mid \pi(b) h\right)=(x \otimes \pi(b) h \mid x \otimes \pi(b) h)
$$

since $x \cdot b \in \mathbf{X} \cdot J$. Then, using similar calculations, we can see that

$$
\begin{aligned}
\| u_{i} \cdot x \otimes \pi(b) h & -x \otimes \pi(b) h \|^{2} \\
& =\left(u_{i} \cdot x \otimes \pi(b) h-x \otimes \pi(b) h \mid u_{i} \cdot x \otimes \pi(b) h-x \otimes \pi(b) h\right)
\end{aligned}
$$

converges to zero as required.

Proposition 4.9. Suppose that $L$ has kernel $P$ and that $\left.\pi\right|_{A}$ is homogeneous. Then, for all $x \in G, \rho_{x G_{P}}$ is a homogeneous representation of $A$ with kernel $x \cdot P$.

Proof. By Lemma 4.6 and our assumptions on $L$ and $\pi$, we have that $\rho_{e G_{P}}$ is homogeneous with kernel $P$. The rest follows from Lemma 4.7 and Proposition 4.8 .

We have set things up so that the representation of $A$ given by $\left.\hat{\Pi}\right|_{A}$ (where $\hat{\Pi}$ is defined in (4.8)) is the direct integral

$$
\int_{G / G_{P}}^{\oplus} \rho_{x G_{P}} d \bar{\mu}
$$

on $L^{2}\left(G / G_{P} * \mathscr{V}, \bar{\mu}\right)$. Recall that the diagonal operators, $\Delta\left(G / G_{P} * \mathscr{V}, \bar{\mu}\right)$, on $L^{2}\left(G / G_{P} * \mathscr{V}, \bar{\mu}\right)$ are, by definition, the multiplication operators determined by bounded Borel functions on $G / G_{P}$ (see [20, Definition F.13]). In this case,

\footnotetext{
${ }^{3}$ For other such results, see 5 .
} 
$\Delta\left(G / G_{P} * \mathscr{V}, \bar{\mu}\right)$ are exactly the operators of the form $M(\varphi)$ with $\varphi \in L^{\infty}\left(G / G_{P}\right)$ and

where $\varphi \cdot f(x)=\varphi\left(x G_{P}\right) f(x)$.

$$
M(\varphi)(f \otimes \xi)=\varphi \cdot f \otimes \pi
$$

We have worked fairly hard to see that (4.10) is an ideal center decomposition (see [20, Definition G.18 and Theorem G.20]). As in the proof of [2, Theorem 1.7], the essential feature we require from this observation is that $M\left(L^{\infty}\left(G / G_{P}\right)\right)$ lies in the center of the commutant of $\hat{\Pi}(A)$ so that

$$
\hat{\Pi}(A)^{\prime} \subset M\left(L^{\infty}\left(G / G_{P}\right)\right)^{\prime} \subset M\left(C_{0}\left(G / G_{P}\right)\right)^{\prime} .
$$

Lemma 4.10. Let $f \in \Gamma_{c}(G ; \mathscr{B})$ and $\varphi \in C_{c}\left(G / G_{P}\right)$. Then we get a section $f \otimes \varphi$ in $\Gamma_{c}\left(G \times G / G_{P} ; \operatorname{pr}_{1}^{*} \mathscr{B}\right)$ via

$$
f \otimes \varphi(y, x H)=\varphi(x H) f(y) .
$$

Such sections span a dense subspace of $\Gamma_{c}\left(G \times G / G_{P} ; \mathrm{pr}_{1} \mathscr{B}\right)$ in the inductive limit topology.

Proof. Let $\mathcal{G}$ be the span of sections of the form $f \otimes \varphi$. Since $g \cdot(f \otimes \varphi)=(g \cdot f) \otimes \varphi$ and $\psi \cdot(f \otimes \varphi)=f \otimes(\psi \cdot \varphi)$ for $g \in C_{c}(G), f \in \Gamma_{C}(G ; \mathscr{B}), \psi, \varphi \in C_{c}\left(G / G_{P}\right)$, it follows that $\mathcal{G}$ is closed under multiplication by functions in $C_{c}\left(G \times G / G_{P}\right)$.

Notice that, for $(y, x H) \in G \times G / G_{P}$, we have that $\operatorname{pr}_{1}^{*} \mathscr{B}(y, x H)=B(y)$. Therefore, the set $\{f \otimes \varphi(y, x H) \mid f \otimes \varphi \in \mathcal{G}\}$ is dense in $\operatorname{pr}_{1}^{*} \mathscr{B}(y, x H)$. A small variation of [10, Lemma A.4] implies the result.

Proof of Theorem 4.1 in the case $G$ is a group. Suppose $T \in B\left(L^{2}\left(G / G_{P} * \mathscr{V}, \bar{\mu}\right)\right.$ is in the commutant of $\operatorname{Ind}_{G_{P}}^{G} L$. It suffices to see that $T$ is a scalar operator. But $T$ must also commute with $\hat{\Pi}(b)=\left(\operatorname{Ind}_{G_{P}}^{G} L\right)\left(\iota_{\mathscr{B}}(b)\right)$ for all $b \in \mathscr{B}$. In particular, $T$ belongs to $\hat{\Pi}(A)^{\prime}$. Hence $T$ commutes with $M(\varphi)$ for all $\varphi \in C_{c}\left(G / G_{P}\right)$ by (4.11). But

$$
(\mathrm{X}-\mathrm{Ind} L)(f \otimes \varphi)=M(\varphi)\left(\operatorname{Ind}_{G_{P}}^{G} L\right)(f) .
$$

Thus $T$ commutes with $(\mathrm{X}-\mathrm{Ind} L)(f \otimes \varphi)$. By Lemma 4.10 this means $T$ commutes with the irreducible representation $\mathrm{X}-\mathrm{Ind} L$. Thus $T$ is a scalar.

\section{The TYPe I CASE}

In this section, we prove the appropriate analogue of [2, Lemma 3.2]. Recall that if $p: \mathscr{B} \rightarrow G$ is a Fell bundle and if $P$ is a primitive ideal of $A=\Gamma_{0}\left(G^{(0)} ; \mathscr{B}\right)$, then $P \supset I(u)$ for $u=\sigma_{A}(P)$. In particular, we can view $P$ as a primitive ideal of the quotient $A(u)=B(u)$. Of course, $P$ is locally closed in Prim $A$ if and only if it is locally closed when viewed as a primitive ideal of $A(u)$.

Proposition 5.1. Let $p: \mathscr{B} \rightarrow G$ be a separable, saturated Fell bundle over a locally compact groupoid $G$ and let $P$ be a primitive ideal in $\operatorname{Prim} A$ where $A=\Gamma_{0}\left(G^{(0)} ; \mathscr{B}\right)$ is the associated $C^{*}$-algebra. Suppose that $P$ is locally closed in $\operatorname{Prim} A$ and that $L$ is an irreducible representation of $C^{*}\left(G_{P}, \mathscr{B}\right)$ which is the integrated form of the strongly continuous map $\pi:\left.\mathscr{B}\right|_{G_{P}} \rightarrow B(\mathcal{V})$ such that $\left.\operatorname{ker} \pi\right|_{A(u)}=P$ where $u=\sigma_{A}(P)$. Then $\left.\pi\right|_{A(u)}$ is a homogeneous representation of $A(u)$.

We'll need the following standard observation from Morita theory.

Lemma 5.2. Suppose that $\mathrm{X}$ is an $A-B$-imprimitivity bimodule. Then the Rieffel correspondence $h: \mathscr{I}(B) \rightarrow \mathscr{I}(A)$ preserves arbitrary intersections. 
Proof. Notice that

$$
h(I)=\{a \in J: a \cdot \mathrm{X} \subset \overline{\mathrm{X} \cdot I}\} .
$$

Hence the result follows once we establish that

$$
\bigcap \overline{\mathrm{X} \cdot I_{J}}=\overline{\mathrm{X} \cdot \bigcap I_{J}}
$$

But this can be proved exactly as in the proof of [20, Lemma 5.19].

Proof of Proposition 5.1. We may as well replace $\mathscr{B}$ by $\left.\mathscr{B}\right|_{G_{P}}$ and $A$ by $A(u)$. Let $\rho=\left.\pi\right|_{A}$. As in the proof of [2, Lemma 3.2], it will suffice to see that if $I$ is any ideal in $A$ such that $I \not \subset P$, then $\overline{\rho(I) \mathcal{V}}=\mathcal{V}$. Since $\rho(I)=\rho(I+P)$, we can assume $I$ properly contains $P$. If $x \in G_{P}$, let $x \cdot I:=h_{x}(I)$. Then $x \cdot I$ properly contains $P$. Since any ideal in $A$ is the intersection of those primitive ideals which contain it,

$$
K:=\bigcap_{x \in G_{P}} x \cdot I \supset \bigcap_{\substack{P^{\prime} \in \operatorname{Prim} \\ P^{\prime} \supset P \\ P^{\prime} \neq P}} P^{\prime}:=J .
$$

Since $P$ is locally closed in Prim $A$, 2, Lemma 3.1] implies that $J$ properly contains $P$. Hence so does $K$. Moreover $K$ must be $G_{P}$-invariant as defined in [7, §3.1]: if $P^{\prime}$ is a primitive ideal containing $K$, then using Lemma 5.2, we have

$$
y \cdot P^{\prime}=h_{y}\left(P^{\prime}\right) \supset \bigcap_{x \in G_{P}} h_{y}(x \cdot I)=\bigcap_{y \in G_{P}} h_{x y}(I)=K .
$$

Thus we can identify $C^{*}\left(G_{P}, \mathscr{B}_{K}\right)$ with an ideal of $C^{*}\left(G_{P}, \mathscr{B}\right)$ as in [7, Lemma 3.5]. We claim

$$
L\left(C^{*}\left(G_{P}, \mathscr{B}_{K}\right)\right) \mathcal{V} \subset \overline{\pi(K) \mathcal{V}}
$$

To see this, let $w \in(\pi(K) \mathcal{V})^{\perp}$. Then for any $h \in \mathcal{V}$,

$$
(L(f) h \mid w)=\int_{G_{P}} \Delta_{G_{p}}(s)^{-\frac{1}{2}}(\pi(f(s)) h \mid w) d s .
$$

But $f(s) \in B(s) \cdot K=(s \cdot K) \cdot B(s)=K \cdot B(s)$. Hence $\pi(f(s)) h \in \pi(K) \mathcal{V}$ for each $h$. Thus the integrand in (5.2) is zero and $(L(f) h \mid w)=0$ for all $w \in(\pi(K) \mathcal{V})^{\perp}$. Thus (5.1) follows.

Next we claim that

$$
L\left(C^{*}\left(G_{P}, \mathscr{B}_{K}\right)\right) \mathcal{V} \neq\{0\} .
$$

To see this, note that as $K$ properly contains $P$, there is an $a \in K$ and $h \in \mathcal{V}$ such that $\pi(a) h \neq 0$. But as Fell bundles always have sufficiently many sections, there is an $f \in \Gamma_{c}\left(G_{P} ; \mathscr{B}_{K}\right)$ such that $f(e)=a$. Since $\pi$ is strongly continuous, there is a neighborhood $V$ of $e$ in $G_{P}$ such that for all $s \in V$ we have

$$
\|\pi(f(s)) h-\pi(a) h\|<\frac{1}{4}\|\pi(a) h\|
$$

and such that

$$
\Delta_{G_{P}}(s)^{-\frac{1}{2}} \leq 2 .
$$

Let $\varphi \in C_{c}^{+}\left(G_{P}\right)$ be such that $\operatorname{supp} \varphi \subset V$ and

$$
\int_{G_{P}} \Delta_{G_{P}}(s)^{-\frac{1}{2}} \varphi(s) d s=1 .
$$


Then $\varphi \cdot f \in \Gamma_{c}\left(G_{P} ; \mathscr{B}_{K}\right)$ and

$$
\begin{aligned}
\|L(\varphi \cdot f) h-\pi(a) f\| & =\left\|\int_{G_{P}} \Delta_{G_{P}}(s)^{-\frac{1}{2}} \varphi(s)(\pi(f(s)) h-\pi(a) h) d s\right\| \\
& <2 \frac{1}{4}\|\pi(a) h\|=\frac{1}{2}\|\pi(a) h\| .
\end{aligned}
$$

Thus $L(\varphi \cdot f) h \neq 0$. This establishes (5.3).

However, as $L$ is assumed irreducible, (5.3) and (5.1) imply that

$$
\overline{L\left(C^{*}\left(G_{P}, \mathscr{B}_{K}\right)\right) \mathcal{V}}=\mathcal{V} \subset \overline{\pi(K) \mathcal{V}} .
$$

Thus $\overline{\rho(I) \mathcal{V}}=\mathcal{V}$ and $\rho$ is homogeneous as claimed.

Proposition 5.1 and Theorem 4.1 imply immediately the following generalization of [2, Theorem 1.8].

Proposition 5.3. Suppose that $p: \mathscr{B} \rightarrow G$ is a separable, saturated Fell bundle over a locally compact groupoid $G$ such that all points are locally closed in Prim $A$. Then $p: \mathscr{B} \rightarrow G$ satisfies strong-EHI. In particular, if $A$ is of type $I$, then $p: \mathscr{B} \rightarrow$ $G$ satisfies the strong-EHI.

Remark 5.4. Even if $A$ is not of type I, there are a number of general situations where Prim $A$ necessarily has locally closed points. For example, as we observe in [2, Proposition 1.8], this is the case in any subquotient of the group $C^{*}$-algebra of an almost connected Lie group. Nevertheless, there are separable $C^{*}$-algebras for which this property fails (see [2, Example 3.3]).

\section{EXAMPLES}

6.1. Groupoid dynamical systems. Let $G$ be a locally compact Hausdorff groupoid with Haar system $\left\{\lambda^{u}\right\}_{u \in G^{(0)}}$. Let $\pi: \mathscr{A} \rightarrow G^{(0)}$ be an upper semicontinuous $C^{*}$-bundle over $G^{(0)}$ and let $A=\Gamma_{0}\left(G^{(0)}, \mathscr{A}\right)$. Assume that $(\mathscr{A}, G, \alpha)$ is a groupoid dynamical system (see, for example, [11, Definition 4.1]). Recall from $[10, \S 2]$ that one can define a Fell bundle $p: \mathscr{B} \rightarrow G$, where $\mathscr{B}:=r^{*} \mathscr{A}=\{(a, x)$ : $\pi(a)=r(x)\}$ is the pull-back of $\mathscr{A}$ via the range map $r$. The multiplication on $\mathscr{B}$ is defined via

$$
(a, x)(b, y):=\left(a \alpha_{x}(b), x y\right),
$$

if $(x, y) \in G^{(2)}$, and the involution is given by

$$
(a, x)^{*}:=\left(\alpha_{x}^{-1}\left(a^{*}\right), x^{-1}\right) .
$$

Then $\Gamma_{c}\left(G, r^{*} \mathscr{A}\right)$ is a $*$-algebra with respect to the operations

$$
f * g(x)=\int_{G} f(y) \alpha_{y}\left(g\left(y^{-1} x\right)\right) d \lambda^{r(x)}(y) \quad \text { and } \quad f^{*}(x)=\alpha_{x}\left(f\left(x^{-1}\right)^{*}\right) .
$$

The crossed product $\mathscr{A} \rtimes_{\alpha} G$ is the enveloping $C^{*}$-algebra of $\Gamma_{c}\left(G ; r^{*} \mathscr{A}\right)$.

Recall (see, for example, [11, Definition 7.5]) that a unitary representation of a groupoid $G$ with Haar system $\left\{\lambda^{u}\right\}_{u \in G^{(0)}}$ is a triple $\left(\mu, G^{(0)} * \mathcal{H}, L\right)$ consisting of a quasi-invariant measure $\mu$ on $G^{(0)}$, a Borel Hilbert bundle $G^{(0)} * \mathcal{H}$ over $G^{(0)}$ and a Borel homomorphism $\tilde{U}: G \rightarrow \operatorname{Iso}\left(G^{(0)} * \mathcal{H}\right)$ such that

$$
\tilde{U}(x)=\left(r(x), L_{x}, s(x)\right),
$$

where $\operatorname{Iso}\left(G^{(0)} * \mathcal{H}\right)$ is the isomorphism groupoid. 
A covariant representation $\left(M, \mu, G^{(0)} * \mathcal{H}, U\right)$ of $(\mathscr{A}, G, \alpha)$ ([11, Definition 7.9]) consists of a unitary representation $\left(\mu, G^{(0)} * \mathcal{H}, U\right)$ of $G$ and a $C_{0}\left(G^{(0)}\right)$-linear representation $M: A \rightarrow B\left(L^{2}\left(G^{(0)} * \mathcal{H}, \mu\right)\right)$ such that there are representations $M_{u}: A \rightarrow B(\mathcal{H}(u))$ so that

$$
M(a) h(u)=M_{u}(a)(h(u)) \text { for } \mu \text {-almost all } u,
$$

and such that there is a $\nu:=\lambda \circ \mu$-null set $N$ such that for all $x \notin N$,

$$
U_{x} M_{s(x)}(b)=M_{r(x)}\left(\alpha_{x}(b)\right) U_{x} \text { for all } b \in A(s(x)) .
$$

Recall from [11, Proposition 7.11] that if $\left(M, \mu, G^{(0)} * \mathcal{H}, U\right)$ is a covariant repre-

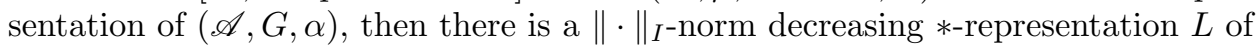
$\Gamma_{c}\left(G ; r^{*} \mathscr{A}\right)$ called the integrated form of the covariant representation given by

$$
L(f) h(u)=\int_{G} M_{u}(f(x)) U_{x} h(s(x)) \Delta(x)^{-\frac{1}{2}} d \lambda^{u}(x) .
$$

Conversely, given any representation $L$ of $\mathscr{A} \rtimes_{\alpha} G$, there is a covariant representation $\left(M, \mu, G^{(0)} * \mathcal{H}, U\right)$ such that $L$ is equivalent to the corresponding integrated form ([11, Theorem 7.12]).

Notice that if $G$ is a group and $(\rho, U)$ is a covariant representation of $(A, G, \alpha)$, then, since we are treating $G$ as a groupoid, the corresponding integrated form is given by the formula

$$
\rho \rtimes U(f)=\int_{G} \rho(f(s)) U_{s} \Delta(s)^{-\frac{1}{2}} d \mu(s)
$$

(compare this formula against, for example, [20, Equation (2.19)]).

If $H$ is a closed subgroupoid of $G$ and $L$ is a representation of $\mathscr{A} \rtimes_{\left.a\right|_{H}} H$, then the construction of induced representations from [17, §4.1] (see also Section 2 above) gives us that the induced representation $\operatorname{Ind}_{H}^{G} L$ acts on the completion of $X \odot \mathcal{H}_{L}$ by

$$
\left(\operatorname{Ind}_{H}^{G} L\right)(f)(\varphi \otimes h)=f * \varphi \otimes h,
$$

where $f * \varphi(z)=\int_{G} f(y) \alpha_{y}\left(\varphi\left(y^{-1} z\right)\right) d \lambda^{r(z)}(y)$. Our Theorem 2.1 seems to be new for this set-up.

Theorem 6.1. Assume that $(\mathscr{A}, G, \alpha)$ is a groupoid dynamical system. Let $u \in$ $G^{(0)}$ and suppose that $L$ is an irreducible representation of $\mathscr{A} \rtimes_{\left.\alpha\right|_{G(u)}} G(u)$. Then $\operatorname{Ind}_{G(u)}^{G} L$ is an irreducible representation of $\mathscr{A} \rtimes_{\alpha} G$.

Recall from [7, Remark 3] that the $G$ action on Prim $A$ is the same as the usual one: $x \cdot P=\alpha_{x}(P)$. Our main theorem (Theorem 4.1) becomes:

Theorem 6.2. Let $(\mathscr{A}, G, \alpha)$ be a groupoid dynamical system. Let $P \in \operatorname{Prim} A$ and let $(\rho, U)$ be a covariant representation of $\left(A, G_{P}, \alpha_{\left.\right|_{G_{P}}}\right)$. Assume that $\operatorname{ker} \rho=P$ and that $\rho \rtimes U$ is irreducible. If either $A$ is type $I$ or if $\rho$ is homogeneous, then $\operatorname{Ind}_{G_{P}}^{G}(\rho \rtimes U)$ is irreducible.

In particular we recover the main result of [2]. 
6.2. Green-Renault's twisted groupoid dynamical systems. Suppose that

$$
G^{(0)} \rightarrow S \stackrel{i}{\rightarrow} \Sigma \stackrel{j}{\rightarrow} G \rightarrow G^{(0)}
$$

is a groupoid extension of locally compact groupoids over $G^{(0)}$ where $S$ is a group bundle of abelian groups admitting a Haar system. We view $S$ as a closed subgroupoid of $\Sigma$. We assume that we have a groupoid dynamical system $(\mathscr{A}, \Sigma, \alpha)$, so that $\pi: \mathscr{A} \rightarrow G^{(0)}=\Sigma^{(0)}$ is an upper semicontinuous $C^{*}$-bundle. We also need an element $\chi \in \prod_{s \in S} M(A(r(s)))$ such that

$$
(s, a) \mapsto \chi(s) a
$$

is continuous from $S * \mathscr{A}$ to $\mathscr{A}$, and such that

$$
\alpha_{s}(a)=\chi(s) a \chi(s)^{*} \text { for all }(s, a) \in S * \mathscr{A}
$$

and

$$
\chi\left(\sigma s \sigma^{-1}\right)=\bar{\alpha}_{\sigma}(\chi(s)) \text { for }(\sigma, s) \in \Sigma^{(2)} .
$$

Following [14] and [15], we call $(G, \Sigma, \mathscr{A})$ a twisted groupoid dynamical system. As in [10, Example 2.5], we define an $S$-action on $r^{*} \mathscr{A}=\{(a, \sigma): \pi(a)=r(\sigma)\}$ by

$$
(a, \sigma) \cdot s:=\left(a \chi(s)^{*}, s \sigma\right) .
$$

The associated Fell bundle is then $\mathscr{B}:=r^{*} \mathscr{A} / S$ with the map $p: \mathscr{B} \rightarrow G$, $p([a, \sigma])=j(\sigma)$. The operations in $\mathscr{B}$ are defined via (see [10. Example 2.5] for details):

if $(j(\sigma), j(\tau)) \in G^{(2)}$ and

$$
[a, \sigma][b, \tau]:=\left[a \alpha_{\sigma}(b), \sigma \tau\right]
$$

$$
[a, \sigma]^{*}=\left[\alpha_{\sigma}^{-1}\left(a^{*}\right), \sigma^{-1}\right] .
$$

To define a section of $\mathscr{B}$, we need a continuous function $f: \Sigma \rightarrow \mathscr{A}$ such that $f(\sigma) \in A(r(\sigma))$ and $f(s \sigma)=f(\sigma) \chi(s)^{*}$ for all $s \in S$ and $\sigma \in \Sigma$ such that $(s, \sigma) \in \Sigma^{(2)}$. The corresponding section is given by $\check{f}(j(\sigma))=[f(\sigma), \sigma]$. Replacing $\check{f}$ with $f$, the $*$-operations on $\Sigma$ are given by

$$
f * g(\sigma)=\int_{G} f(\tau) \alpha_{\tau}\left(g\left(\tau^{-1} \sigma\right)\right) d \lambda^{r(j(\sigma))}(\tau) \quad \text { and } \quad f^{*}(\sigma)=\alpha_{\sigma}\left(f\left(\sigma^{-1}\right)^{*}\right) .
$$

As described in [10, Example 2.10], the completion is Renault's $C^{*}(G, \Sigma, \mathscr{A}, \lambda)$ from 14] and [15].

A covariant representation $\left(M, \mu, G^{(0)} * \mathcal{H}, U\right)$ of $(G, \Sigma, \mathscr{A})$ ([14, Definition 3.4]) consists of a unitary representation $\left(\mu, G^{(0)} * \mathcal{H}, U\right)$ of $\Sigma$ and a $C_{0}\left(G^{(0)}\right)$-linear representation of $M: A \rightarrow B\left(L^{2}\left(G^{(0)} * \mathcal{H}\right), \mu\right)$ so that there are representations $M_{u}: A \rightarrow B(H(u))$ such that

$$
M(a) h(u)=M_{u}(a)(h(u)) \text { for } \mu \text {-almost all } u,
$$

and such that there is a $\mu$-conull set $V$ such that

$$
U_{x} M_{s(x)}(a)=M_{r(x)}\left(\alpha_{x}(a)\right) U_{x} \text { for all } x \in \Sigma_{V} \text { and } a \in A(s(x)),
$$

and

$$
U_{s}=M_{r(s)}(\chi(s)) \text { for all } s \in S_{V} .
$$

If $\left(M, \mu, G^{(0)} * \mathcal{H}, U\right)$ is a covariant representation of $(G, \Sigma, \mathscr{A})$, then [14, Proposition 3.5] (see also [10, Proposition 4.10]) implies that there is a $\|\cdot\|_{I \text {-norm decreasing }}$ 
*-representation $L$ of $\Gamma_{c}(G, \Sigma, \mathscr{A})$, called the integrated form of the covariant representation, given by

$$
L(f) h(u)=\int_{G} M_{u}(f(x)) U_{x} h(s(x)) \Delta(j(x))^{-1 / 2} d \lambda^{u}(j(x)) .
$$

Conversely, [14, Theorem 4.1] and [10, Theorem 4.13] imply that every representation of $C^{*}(G, \Sigma, \mathscr{A})$ is equivalent to the integrated form of a covariant representation.

Notice that if $\Sigma$ is a group and $S$ is an abelian subgroup of $\Sigma$, then $G=\Sigma / S$. We recover the twisted dynamical systems of Green [4] and Đặng Ngọc [12. If $(\rho, U)$ is a covariant representation of $(A, G, \alpha)$ that preserves $\chi$, i.e. $\bar{\rho}(\chi(s))=U(s)$ for all $s \in S$, then, since we are treating groups as groupoids, the integrated form $\rho \rtimes^{\chi} U$ of $(\rho, U)$ is

$$
\rho \rtimes^{\chi} U(f)=\int_{G} \rho(f(s)) U(s) \Delta(\dot{s})^{-\frac{1}{2}} d \mu(\dot{s}) .
$$

If $H$ is a closed subgroupoid of $G$ and $L$ is a representation of $C^{*}(H, \Sigma, \mathscr{A})$, then the induced representation $\operatorname{Ind}_{H}^{G}$ acts on the completion of $X \odot \mathcal{H}_{L}$ (see Section 2 for details) via

$$
\operatorname{Ind}_{H}^{G}(L)(f)(\varphi \otimes h)=f * \varphi \otimes h,
$$

where $f * \varphi(z)=\int_{G} f(y) \alpha_{y}\left(g\left(y^{-1} z\right)\right) d \lambda^{r(j(z))}(y)$. As an immediate consequence of Theorem 2.1 we obtain the following:

Theorem 6.3. Assume that $(G, \Sigma, \mathscr{A})$ is a twisted groupoid dynamical system. Let $u \in G^{(0)}$ and suppose that $L$ is an irreducible representation of $C^{*}(G(u), \Sigma(u), \mathscr{A})$. Then $\operatorname{Ind}_{G(u)}^{G} L$ is an irreducible representation of $C^{*}(G, \Sigma, \mathscr{A})$.

Using [7, Lemma 2.1] one obtains that the $G$ action on Prim $A$ is the same as the usual one. This fact, together with 4.1 implies the following:

Theorem 6.4. Let $(G, \Sigma, \mathscr{A})$ be a twisted groupoid dynamical system. Let $P \in$ Prim $A$ and let $(\rho, U)$ be a covariant representation of $\left(G_{P}, \Sigma_{P}, \mathscr{A}\right)$ that preserves $\chi$ and is such that $\operatorname{ker} \rho=P$ and $\rho \rtimes^{\chi} U$ is irreducible. If either $A$ is of type $I$ or if $\rho$ is homogeneous, then $\operatorname{Ind}_{G_{P}}^{G} \rho \rtimes^{\chi} U$ is irreducible.

\section{REFERENCES}

[1] Alcides Buss, Ralf Meyer, and Chenchang Zhu, A higher category approach to twisted actions on $C^{*}$-algebras, Proc. Edinb. Math. Soc. (2) 56 (2013), no. 2, 387-426, DOI 10.1017/S0013091512000259. MR3056650

[2] Siegfried Echterhoff and Dana P. Williams, Inducing primitive ideals, Trans. Amer. Math. Soc. 360 (2008), no. 11, 6113-6129, DOI 10.1090/S0002-9947-08-04499-1. MR2425706 (2009g:46130)

[3] Edward G. Effros, A decomposition theory for representations of $C^{*}$-algebras., Trans. Amer. Math. Soc. 107 (1963), 83-106. MR0146682 (26 \#4202)

[4] Philip Green, The local structure of twisted covariance algebras, Acta Math. 140 (1978), no. 3-4, 191-250. MR0493349 (58 \#12376)

[5] Astrid An Huef, Iain Raeburn, and Dana P. Williams, Properties preserved under Morita equivalence of $C^{*}$-algebras, Proc. Amer. Math. Soc. 135 (2007), no. 5, 1495-1503 (electronic), DOI 10.1090/S0002-9939-06-08625-4. MR2276659 (2008d:46071)

[6] Marius Ionescu and Dana P. Williams, Irreducible representations of groupoid $C^{*}$-algebras, Proc. Amer. Math. Soc. 137 (2009), no. 4, 1323-1332, DOI 10.1090/S0002-9939-08-09782-7. MR2465655(2009m:46102)

[7] Marius Ionescu and Dana P. Williams, Remarks on the ideal structure of Fell bundle $C^{*}$ algebras, Houston J. Math. 38 (2012), no. 4, 1241-1260. MR.3019033 
[8] S. Kaliszewski, Paul S. Muhly, John Quigg, and Dana P. Williams, Coactions and Fell bundles, New York J. Math. 16 (2010), 315-359. MR2740580(2012d:46165)

[9] Paul S. Muhly, Bundles over groupoids, Groupoids in analysis, geometry, and physics (Boulder, CO, 1999), Contemp. Math., vol. 282, Amer. Math. Soc., Providence, RI, 2001, pp. 67-82, DOI 10.1090/conm/282/04679. MR1855243 (2003a:46085)

[10] Paul S. Muhly and Dana P. Williams, Equivalence and disintegration theorems for Fell bundles and their $C^{*}$-algebras, Dissertationes Math. (Rozprawy Mat.) 456 (2008), 1-57, DOI 10.4064/dm456-0-1. MR2446021 (2010b:46146)

[11] Paul S. Muhly and Dana P. Williams, Renault's equivalence theorem for groupoid crossed products, NYJM Monographs, State University of New York University at Albany, Albany, NY, 2008, volume 3, Available at http://nyjm.albany.edu:8000/m/2008/3.htm.

[12] Nghiêm Đặng Ngọc, Produits croisés restreints et extensions of groupes, 1977, Notes, Paris.

[13] Iain Raeburn and Dana P. Williams, Morita equivalence and continuous-trace $C^{*}$-algebras, Mathematical Surveys and Monographs, vol. 60, American Mathematical Society, Providence, RI, 1998. MR1634408 (2000c:46108)

[14] Jean Renault, Représentation des produits croisés d'algèbres de groupoïdes (French), J. Operator Theory 18 (1987), no. 1, 67-97. MR.912813 (89g:46108)

[15] Jean Renault, The ideal structure of groupoid crossed product $C^{*}$-algebras, J. Operator Theory 25 (1991), no. 1, 3-36. With an appendix by Georges Skandalis. MR.1191252 (94g:46074)

[16] Jean-Luc Sauvageot, Idéaux primitifs induits dans les produits croisés (French), J. Funct. Anal. 32 (1979), no. 3, 381-392, DOI 10.1016/0022-1236(79)90047-8. MR538862(81a:46080)

[17] Aidan Sims and Dana P. Williams, Renault's equivalence theorem for reduced groupoid $C^{*}$ algebras, J. Operator Theory 68 (2012), no. 1, 223-239. MR2966043

[18] Aidan Sims and Dana P. Williams, Amenability for Fell bundles over groupoids, Illinois J. Math. 57 (2013), no. 2, 429-444. MR3263040

[19] Aidan Sims and Dana P. Williams, An equivalence theorem for reduced Fell bundle $C^{*}$ algebras, New York J. Math. 19 (2013), 159-178. MR 3084702

[20] Dana P. Williams, Crossed products of $C^{*}$-algebras, Mathematical Surveys and Monographs, vol. 134, American Mathematical Society, Providence, RI, 2007. MR2288954 (2007m:46003)

Department of Mathematics, Colgate University, Hamilton, New York 13346

E-mail address: mionescu@colgate.edu

Current address: Department of Mathematics, United States Naval Academy, Annapolis, Maryland 21402

E-mail address: ionescu@usna.edu

Department of Mathematics, Dartmouth College, Hanover, New Hampshire 037553551

E-mail address: dana.williams@Dartmouth.edu 